\title{
CIENCIA Y SABERES LOCALES EN LA POSGUERRA: LA ASOCIACIÓN INTERNACIONAL AMERICANA PARA EL DESARROLLO ECONÓMICO Y SOCIAL (AIA) Y LOS PROGRAMAS DE MODERNIZACIÓN DE LA AGRICULTURA EN BRASIL (1945-1961)
}

\author{
Claiton Marcio da Silva \\ Universidade Federal da Fronteira Sul/Brasil \\ claiton@uffs.edu.br
}

Recibido: 17 octubre 2014; Aceptado: 1 febrero de 2015.

Cómo citar este artículo/Citation: Silva, Claiton Marcio da (2015), “Ciencia y saberes locales en la posguerra: la Asociación Internacional Americana para el Desarrollo Económico y Social (AIA) y los programas de modernización de la agricultura en Brasil (1945-1961)", Asclepio, 67 (1): p082. doi: http://dx.doi.org/10.3989/asclepio.2015.08

RESUMEN: Este artículo discute la actuación de la Asociación Internacional Americana para el Desarrollo Social y Económico (AIA) en Brasil. La acción de la AIA se caracterizó por un intenso proceso de negociación entre 1946 y 1961. Contemporánea de la emergente ideología de la modernización, la AIA estudió los posibles proyectos adecuados para la América Latina, en especial Brasil y Venezuela. Inspirada en las experiencias norteamericanas del crédito rural, el extensionismo, entre otras estrategias de desarrollo, la agencia procuró adaptar estos modelos a las condiciones locales, partiendo de la base de que, desde el punto de vista de la AIA, los proyectos exitosos en Estados Unidos, si fueran adaptados, tendrían también éxito en los países en desarrollo. Sin embargo, las experiencias en la provincia brasileña de Minas Gerais demostraron grandes dificultades de ejecución.

PALABRAS CLAVE: Agricultura; Ciencia; Modernización; Brasil; Posguerra.

SCIENCE AND LOCAL KNOWLEDGE IN POST WAR: THE AMERICAN INTERNATIONAL ASSOCIATION (AIA) AND THE MODERNIZATIONS OF AGRICULTURE IN BRAZIL (1945-1961)

ABSTRACT: It aims to discuss the work of American International Association for Economic and Social Development (AIA) in Brazil. AlA's work was characterized for a intense process of negotiation with local governments between 1946 and 1961 . Contemporary to the rise of the ideology of modernization, AIA introduced some projects which were considered suitable for Latin America, as Brazil and Venezuela in special. Following the American experiences in supervised credit and extension work, this agency tried to adapt this models to local conditions, interpreting that successful projects earlier developed in United States could be also successful in Latin America. On the other hand, these set of experiences in Brazilian states like Minas Gerais had strong difficulties to be developed.

KEY WORDS: Agriculture; Science; Modernization; Brazil; Post-war times. 


\section{INTRODUCCIÓN}

Las interpretaciones acerca de la agricultura y del medio rural en Brasil, desde los tiempos de la Colonia Portuguesa (1500 a 1822), del Imperio (1822 a 1889) y de la República (1889), hasta llegar a la Segunda Guerra Mundial (1945), se destacan por diferentes proposiciones, aunque con una característica común: el retraso. Según algunos políticos, intelectuales, agrónomos y agricultores prácticos, la herencia portuguesa en la agricultura brasileña estaría amparada en características como la devastación ambiental, el latifundio, las prácticas agrícolas primitivas y la agricultura extensiva. Sumado a estas drásticas características, está el hecho de que la población mestiza tenía poca adhesión al trabajo rutinario, predominando la herencia indígena y afrodescendiente, orientada sobre todo a la subsistencia. Consecuentemente, aunque los recursos naturales eran y siguen siendo abundantes, la población rural y las técnicas agrícolas adoptadas a lo largo de los años no coinciden con los proyectos de desarrollo de la nación.

Con la creación de espacios de ciencia, a partir del período del Brasil Imperio, entre 1822 y 1889 (intervalo entre su independencia de Portugal y la conformación de República que es hoy), la tónica modernizante pasó a constituir parte del vocabulario de los pensadores de la agricultura en Brasil, aunque los proyectos para alcanzar tal modernización hayan sido divergentes entre si. Los paradigmas europeos, principalmente el francés y el alemán, dominarían el discurso durante las primeras décadas del siglo XX, dominando, sobre todo, en el ámbito de la Escuelas Superiores Agrícolas. Luego, la experiencia de los norteamericanos alcanzaría todo el territorio nacional, reemplazando el modelo europeo.

El argumento central de este artículo aborda como el proceso de americanización de la agricultura ganó espacio como consecuencia del alineamiento político entre Brasil y Estados Unidos durante la Segunda Guerra Mundial. En su implementación a lo largo de los años cincuenta, sin embargo, el contexto local favoreció la reorientación de diversos proyectos. Así, este texto problematiza el proceso de construcción de un modelo hegemónico para la agricultura brasileña, iniciado con la actuación de agencias internacionales y más tarde institucionalizada por el Estado Brasileño. De este modo, se busca evidenciar las relaciones de poder presentes en los organismos de decisión política, la inserción de la agronomía en este proceso y el vínculo con los agricultores brasileños.
Para ello, el presente artículo está dividido en tres secciones que abordan: 1) las relaciones políticas entre Brasil y Estados Unidos; 2) la actuación de los dirigentes políticos, agrónomos y las experiencias de modernización de la agricultura en la década de 1950; y 3) los impactos para los agricultores y rumbos posteriores a la Segunda Guerra Mundial.

\section{BRASIL Y ESTADOS UNIDOS: COOPERACIÓN TÉCNICA EN AGRICULTURA POS 1945}

El mundo de mañana, brillante con la promesa de una vida mejor necesita nuevas carreteras para el avance de la ciencia y la tecnología a través de los obstáculos de la lengua, la raza y las costumbres. AIA es una manera de estrechar estas brechas entre las personas para que los beneficios de la ciencia y las nuevas tecnologías puedan propagarse más ampliamente sobre la tierra.

Nelson Rockefeller

La afirmación de Nelson Rockefeller sobre la American International Association for Economic and Social Development (AIA) - la agencia filantrópica ideada por él mismo y por algunos de sus compañeros de los tiempos de guerra - refleja presupuestos característicamente iluministas, dentro de los cuales la ciencia y la tecnología serían consideradas las formas más adecuadas de llevar sus progresos a las más diferentes naciones, junto a eso, a una forma de acción característica de la post Segunda Guerra. El antropólogo colombiano Arturo Escobar sostiene que especialistas de las Naciones Unidas volcados en el objetivo de diseminar políticas económicas promulgaban en 1951 que el progreso económico no sería posible sin que las viejas instituciones sociales, las diferencias de clase, raza y credo fuesen desechas (Escobar, 1995, p. 20).

En algunas circunstancias, el liberal e internacionalista, Nelson Rockefeller atendía a impulsos semejantes a los de los críticos del New Deal y aislacionistas, como el conservador y senador Republicano Kenneth Wherry. De acuerdo, con el historiador Michael E. Latham, Wherry durante la guerra fue un "proponente típico de una campaña para modernizar el mundo" a la imagen de los Estados Unidos (Latham, 2003, p. 1). De cierta forma, Wherry no era el único en pensar que el liberalismo, el capitalismo y la democracia deberían corresponder a un grado para ser alcanzado por otras sociedades. Muchos investigadores asumieron esta concepción de modernización como un instrumento político, como Kenneth Kadow, socio de Rockefeller en la creación de la AIA, en 1946 le escribía en la misma línea: "Si nosotros en los Estados 
Unidos creemos en la economía, política, filosofías sociales y prácticas que nos han hecho una gran nación, es nuestro deber y el privilegio de hacer todo lo que podamos de una manera concreta y práctica para trabajar con otros pueblos del mundo hacia los logros de objetivos similares o igualmente satisfactorios" (AIA, 1946, p. 14) Sin embargo, si el énfasis de Wherry recayó sobre China, Nelson Rockefeller, Kenneth Kadow y los demás miembros de la AIA trataron de orientar sus acciones hacia la América Latina.

La AIA trajo para el contexto post-1945 acciones que, de alguna forma, estaban siendo puestas en práctica desde las décadas de 1920 y 1930. Según el historiador Michael Adas, aunque el término "modernización" fuera raramente utilizado antes del final de la Segunda Guerra Mundial, tarea semejante fue realizada por misioneros, educadores e ingenieros que promovieron cambios culturales, políticos y económicos en China, Filipinas y América Latina en aquellas décadas (Adas, 2003 , p. 26). En los primeros años del siglo XX, de acuerdo con el historiador Thomas O'Brien, la actuación de las corporaciones norteamericanas en países de Latinoamérica dejó su marca en las relaciones entre el sector público - representado por las elites latinoamericanas - y la iniciativa privada, influyendo en este proceso de modernización (O’Brien, 1996, p. 2-4).

En este sentido, la AIA ejerció una especie de vanguardia en la posguerra al emprender un tipo de cooperación técnica pautada en principios que serían institucionalizados en programas de asistencia técnica como, por ejemplo, el Point Four ${ }^{1}$ del gobierno de Harry Truman. El discurso inaugural del referido presidente el 20 de enero de 1949, estuvo marcado por el entendimiento de que sería necesario que los Estados Unidos fuesen un elemento activo en el auxilio a la modernización de los demás países, conllevando la industrialización, urbanización, crecimiento de la producción material, de los niveles de vida con ideales educacionales y culturales modernos. Otro aspecto relacionado con este ideal fue justamente la necesidad de desarrollar técnicamente la agricultura (Escobar, 1995, p. 20) Sin embargo, si se considera este momento del discurso de Truman un marco fundacional de la difusión de los ideales de modernización, se debe tener en cuenta que agencias como la AIA ya desarrollaban estas premisas desde 1946.

En las décadas de 1950 y 1960, en el contexto de la Guerra Fría, las proposiciones del Point Four y posteriormente de la Teoría de la Modernización se tornaron un poderoso modelo de análisis y prescripción. Para Michael Latham, la Teoría de la Modernización se ha convertido en la más influyente manera de entender el mundo y direccionar su futuro (Latham, 2003, p. 18). A diferencia de la ideología imperialista del giro del siglo XIX o del Destino Manifiesto, las acciones de agencias internacionales como la AIA, estarían más próximas a un discurso benevolente. En este contexto, la participación de las agencias se caracterizaría no solo por la simple distinción de recursos procedentes de las colonias para los países colonizadores sino que de la ciencia y de la tecnología y todos los beneficios que deberían ser distribuidos "ampliamente en la tierra", de acuerdo con las palabras del propio Rockefeller.

La utilización de estos beneficios como instrumento político efectivo - una ideología acorde con la sugerencia del historiador Michael Latham - prometía encontrar la manera más adecuada para que las visiones de cambios sociales y progreso pudiesen garantizar un nuevo orden, y, con eso, los Estados Unidos dispondrían en los países en proceso de descolonización o desarrollo, un modelo involucrando una determinada concepción del capitalismo, liberalismo y democracia (Latham, 2003, p. 18). Todo esto, junto a la idea de modernización, incluso aquella aplicada a la agricultura y al medio rural en los países de Latinoamérica. Así se discutirá cómo los principales actores de este proceso establecieron procesos de negociación, involucrando agencias internacionales, políticos y también agentes de modernización como, por ejemplo, los ingenieros agrónomos.

\section{DIRIGENTES POLÍTICOS, AGRÓNOMOS Y ALGUNAS EXPERIENCIAS DE MODERNIZACIÓN DE LA AGRICUL- TURA BRASILEÑA EN LA DÉCADA DE 1950}

Este apartado explora las formas en que se desarrollarían los principales programas de cooperación técnica entre la AIA y las instituciones brasileñas. Entre 1946 y 1950, se destacan las actividades vinculadas al Serviço de Alimentação da Previdência Social - (SAPS) ${ }^{2}$. No obstante, el foco de atención se centra en las dos principales experiencias con participación de la AIA: la primera de ellas se realizó en el interior de la provincia de São Paulo, en las ciudades de Santa Rita do Passa Quatro y de São José do Rio Pardo, iniciadas formalmente en 1949 y 1950, respectivamente y finalizadas a mediados de 1956; la otra experiencia se considera la de mayor éxito de la AIA en Brasil, o sea, la creación de la Associação de Crédito e Assistência Rural (ACAR) ${ }^{3}$ en diciembre de 1948, con la cooperación del gobierno de Minas Gerais. La experiencia de la ACAR se expandió en las décadas siguientes por todo el territorio nacional, durante y después del gobierno de Juscelino Kubitschek (1956-1961). 
Figura 1. Las agencias de cooperación técnica de Nelson Rockefeller en Brasil (1941-1956)

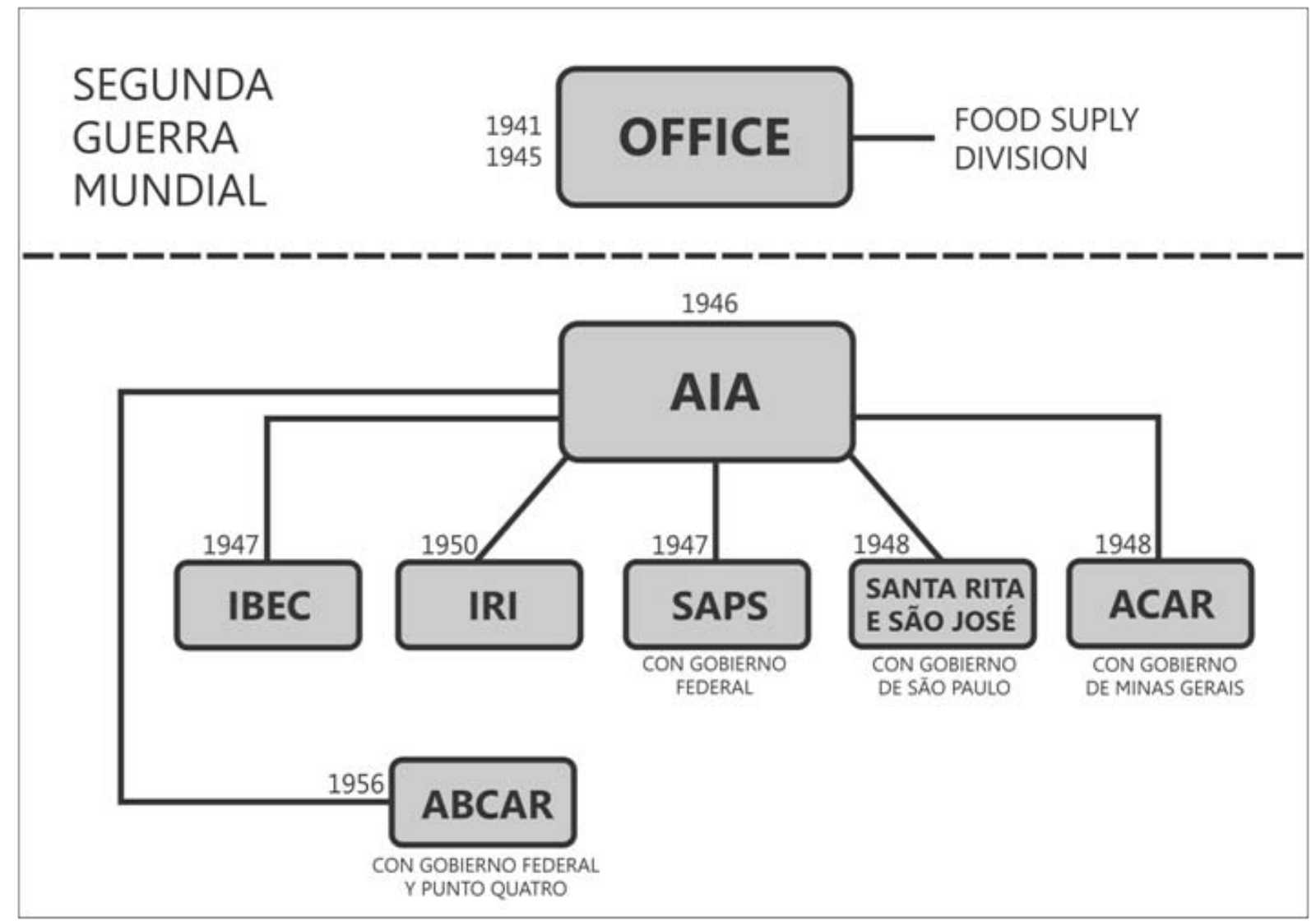

Elaboración del autor con la colaboración de Everton Gabriel Bortoletti

Las experiencias del SAPS, de Santa Rita y São José, así como otras pequeñas actividades, generalmente no son abordadas por la producción bibliográfica que trata sobre el tema. Así, un importante momento de la negociación y adecuación entre una agencia internacional e instituciones brasileñas se deja de lado. Como consecuencia, la bibliografía evidencia los consensos establecidos, pero se borra muchas veces el proceso anterior de negociación y los posibles conflictos. Un ejemplo sería el argumento defendido por la periodista directamente vinculada a la AIA, Martha Dalrymple, que es retomada por otros autores, como Cary Reich y José Paulo Ribeiro. Todos ellos sostienen la aceptación de representantes brasileños con relación a las "ideas de Nelson Rockefeller" (Dalrymple, 1968; Reich, 1996 y Ribeiro, 2000).

En este apartado, se analizan las negociaciones entre la AIA y las administraciones provinciales o municipales en dos provincias brasileñas, São Paulo y Minas Gerais: en la primera provincia, los programas desarrollados en dos ciudades, Santa Rita do Passa
Quatro y São José do Rio Pardo, se proponían articular tanto la administración provincial como la municipal, y también hacendados y empresarios. En definitva, era un acuerdo con el ayuntamiento de estos municipios que se prorrogó posteriormente, requiriendo el apoyo de la administración provincial. Por su parte, Minas Gerais inició su programa de manera inversa, o sea, constituyendo un programa a nivel provincial y buscando ampliar cada año geograficamente su radio de actuación con el apoyo de los ayuntamientos. Otra diferencia reside en el hecho de que en el interior de la provincia de São Paulo, la actuación de los programas se realizó por una oficina de la AIA, que reunía los recursos de la agencia filantrópica y contaba con el apoyo de administraciones provinciales, municipales y de empresarios. En Minas Gerais, una agencia compuesta de una Junta de Administración mixta, involucrando tanto técnicos norteamericanos como brasileños, pero, también, políticos brasileños, se organizó y reunió los esfuerzos de la AIA y gubernamentales para la modernización de la agricultura. 
La experiencia paulista (de São Paulo) se ha limitado a la instalación de dos oficinas en las ciudades citadas y finalizó sus actividades en 1956 por falta de apoyo financiero y político. Minas Gerais, empero, inició con un pequeño programa que fue ampliado, alcanzando la mayor parte del territorio del estado al inicio de la década de 1960.

Si las experiencias paulista (de São Paulo) y mineira (de Minas Gerais) de actuación conjunta entre la AIA y los gobiernos locales poseen semejanzas en lo que se refiere a la adopción de prácticas involucradas en la agricultura, también se sabe que el proyecto mineiro se llevó adelante en el sentido de la nacionalización durante el gobierno del presidente Juscelino Kubitschek (1956-1961). Con vistas a exponer el programa paulista para, enseguida, abordar las posibles diferencias y semejanzas entre los programas, este tópico discutirá como existía una diferencia entre la visión de trabajo de la AIA y de los gobiernos locales que resultó en la extinción del programa en 1956. En otras palabras, la elite política de la provincia de São Paulo entendía que la estructura de investigación agrícola existente desde antaño ofrecía condiciones para emprender un proceso de modernización de la agricultura; sin embargo, la AIA legitimaba su actuación tratando de demostrar que existían diversos problemas para solucionar desde la cuestión de un posible agotamiento de los recursos naturales hasta las técnicas y tecnologías aplicadas al proceso de producción agrícola.

No obstante, los discursos de la AIA y de los representantes locales se asemejaban durante los primeros años de los programas desarrollados en Santa Rita do Passa Quatro y en São José do Rio Preto, principalmente en el gobierno de Adhemar de Barros (1947-1951) y en parte del período en que Lucas Garcez (1951-1955) estuvo frente al poder ejecutivo provincial (Cotta, 2008, p. 45). En tanto, los proyectos de expansión e intensificación de estos programas van alejando a los actores, haciendo que la actuación de la AIA se centre cada vez más en la provincia de Minas Gerais, mientras que los paulistas se apoyan en sus programas preexistentes ${ }^{4}$.

La conclusión de un programa experimental de la AIA, intitulado Livestock and parasite control investigations and demonstrationsy, que actuaba en diversas provincias, entre los cuales figuran São Paulo y Minas Gerais, garantizó que la continuidad de estas actividades fueran realizadas por otro proyecto, el de número 8 , intitulado Brazil, community services program. Este proyecto se inició en Santa Rita do Passa Quatro, pero también originó el acuerdo con el gobierno de Minas
Gerais, que a su vez posibilitó la reacción de la ACAR el 6 de diciembre de 1948. A pesar de que en 1946 la AIA estableció una oficina central en la provincia de São Paulo, administrada por Robert Hudgens y teniendo como principal responsable técnico el norteamericano John B. Griffing, que dirigió la Escola Superior de Agricultura e Veterinária, en Viçosa (Minas Gerais), entre 1936 y 1939. El 1 de agosto de 1948 se iniciaron las negociaciones entre la AIA y el ayuntamiento de Santa Rita con la intención de establecer un proyecto cooperativo. Las negociaciones involucraron no solo el alcalde sino que también los hacenderos de la región. Con estrategia de convencimiento, la AIA realizó ensayos demostrativos (demonstration work) supervisando el control de las garrapatas bovinas el 29 de agosto de 1948; pocos días después, el 4 de septiembre, se firmó un acuerdo de asistencia técnica entre la agencia, el ayuntamiento de Santa Rita y los hacendados locales (AIA, 1949, s. p.), incluyendo, posteriormente, otras agencias.

Con relación a las negociaciones políticas, si el inicio de la década de 1950 fue marcado por la posibilidad de consonancia de proyectos, a la mitad de la misma década los anhelos del AIA y de los políticos del estado se distanciaron. Por ejemplo, el 21 de diciembre de 1951 la AIA firmó un convenio con las Secretarías de Educación, Salud y Agricultura de la provincia de São Paulo para dar continuidad a los programas de cooperación técnica en los municipios de Santa Rita y São José (From Walter Crawford to Wallace Harrison, 1954 , p. 1). Previsto hasta el 31 de diciembre de 1956, el acuerdo dejaría para la Secretaría de Agricultura la responsabilidad de realizar proyectos como clubes de economía doméstica e implementación de silostrinchera. De la misma manera, las demás secretarías mantendrían los proyectos, $y$, tal como el primer proyecto, deberían posibilitar un proceso de expansión de estas actividades para otros municipios de la provincia. A su vez, la AIA dispondría la orientación técnica para las actividades agrícolas desarrolladas en los dos municipios, así como, en otros, si se ampliase el área de actuación.

Con esto, la AIA podría diseminar y orientar en otros municipios las "técnicas modernas de agricultura como aquellas demostradas en Santa Rita y São José". Las actividades se mantendrían por la AIA por tiempo suficiente hasta que la Secretaría de Agricultura pudiese asumir las responsabilidades de la continuidad del proyecto. Sin embargo, el 11 de mayo de 1954, Mr. Crawford declaró: "las agencias de la provincia no han cumplido con su parte del acuerdo, que era para 
expandir el trabajo hecho por la AIA en Santa Rita y São José hasta otros veinte municipios". Tampoco "hay otros municipios de que serán capaces de hacerIo". En función de esto, Crawford negoció para que los programas fuesen realizados por las tres secretarías mencionadas, solo por un periodo de dos años (From Walter Crawford to Wallace Harrison, 1954, p. 1). Incluso colaboradores de Nelson Rockefeller, como Renato Costa Lima, crearon dificultades para los trabajos de la AIA, de acuerdo con João Napoleão Andrade (Andrade, 1982, p. 29).

De esta forma, si el gobierno de São Paulo no demostraba actitudes de apoyo conforme el deseo de la AIA, los miembros brasileños de la ACAR y el gobierno de Minas Gerais estaban "ansiosos por continuar y ampliar la labor" (From Walter Crawford to Wallace Harrison, 1954, p. 2). Así, propondrían, la apertura de diez nuevas oficinas locales y una oficina regional por año. En consecuencia a eso, la actuación de la AIA en la mitad de la década de 1950, estaba cada vez más direccionada para que los gobiernos locales cuidasen los proyectos iniciados por la agencia, principalmente en São Paulo. También, con la reducción del presupuesto para 1955-56, la acción de la AIA estaría posiblemente más concentrada en actividades que pudiesen llevar adelante sus objetivos de desarrollo. Aguardando por la aprobación del acuerdo, Renato Costa Lima sugirió que las operaciones de la AIA siguiesen adelante en conjunto con la Secretaría de Agricultura. Según Henry Bagley, no obstante, para la AIA, la entrada de un nuevo gobernador en las elecciones y la nueva composición de la Asamblea Legislativa dejarían dudas en cuanto a la continuidad del proyecto (From Henry Bagley, 1955, p. 1).

Como forma de resaltar los resultados de su trabajo, la AIA necesitaba apoyo político adecuado para demostrar la real diferencia entre tradicional y moderno. Y, el programa de Minas Gerais traería mayores resultados, haciendo que en 1956, refiriéndose a los programas paulistas, la AIA "no quería poner más dinero en ellos" (From Henry Bagley, 1955, p. 1). Al contrario de la imagen de São Paulo, en cuanto la provincia brasileña más desarrollada económicamente, Minas Gerais, en cambio, se amparaba simbólicamente en una autorepresentación de "estagnación económica", y los vientos de la industrialización que soplaban con uno de los presidentes de Minas, João Pinheiro (1890, 1906-1908), fueron retomados con mayor intensidad con el fin de la Segunda Guerra Mundial, articulando la agricultura a la necesidad de crecimiento industrial. Como ejemplo, el Gobierno de Milton Campos (1947-
1950) desarrolló actividades relacionadas con la producción agrícola y de investigación como la institución de la Universidade Federal de Viçosa (antigua Escola Superior de Agricultura e Veterinária, la ESAV), la Escola Média de Agricultura, entre otras. Otra iniciativa fue el establecimiento del Ensino Agrário Ambulante, durante el gobierno de Milton Campos, que "desarrolló trabajo discreto, pero eficiente, yendo a las más lejanas regiones del estado, llevando al medio rural grande cantidad de pequeños implementos agrícolas, nociones técnicas de agricultura y pecuaria, películas educativas, medicinas y asistencia médica" (Dulci, 1999, p 78). Sin embargo, actividades semejantes fueron desarrolladas por la ACAR que, en poco tiempo tuvo un mayor alcance que el Ensino Agrario Ambulante. Por lo tanto, la ACAR representó la necesidad de las elites mineiras al llevar adelante un proceso de industrialización que estaba mejor consolidado en São Paulo. Este asunto será abordado enseguida.

Si un análisis tradicional puede apuntalar que el proceso de modernización agrícola es externo, o sea, inicial con el acuerdo de la AIA y el Gobierno de la Provincia de Minas Gerais, la discusión aquí propuesta parte del principio que, al revés, existían procesos de modernización de la agricultura iniciados en Minas Gerais - con énfasis en la industrialización - durante la primera mitad del siglo XX. Una de estas experiencias, de la segunda mitad de la década de 1920, fue realizada en la ESAV, que vendría a ser llamada posteriormente de Universidade Federal de Viçosa. El proceso de industrialización, por el cual se entiende también el papel de la agricultura en este proceso, fue desarrollado a lo largo de las primeras décadas del siglo XX. Así, el argumento de este trabajo apunta que la propuesta de Nelson Rockefeller no era totalmente rara al escenario político y económico mineiro.

La articulación entre agricultura e industria en Minas Gerais encuentra en las diversas corrientes políticas de la provincia una forma de explicación. Entonces, de forma subyacente a esas corrientes, se podría pensar en tradición agraria mineira, que de acuerdo con José Murilo de Carvalho, "puede ser vista como ibérica": "tomándose el vocablo como significado, en el mundo privado, apego a la tradición, a la jerarquía, a la religión, a la familia, a la moderación, al trabajo." Estas representaciones persistentes en lo que se refiere principalmente al mundo rural mineiro, son complementadas con otras características en el espacio público, aún de acuerdo con el autor: "aceptación de papel predominante en el Estado en relación a la iniciativa individual, énfasis en la cooperación en oposi- 
ción a la competición, aversión al conflicto, tendencia para resolver las divergencias por arreglos consensuados, preferencia para la conservación en vez de cambio." (Carvalho, 2005, pp. 65-67). Representantes de esta tendencia, predominante entre la segunda mitad del siglo XIX y primera mitad del siglo XX, serían Silviano Brandão, Venceslau Brás y Bias Fortes. Con ellos, el mantenimiento del orden agrario no representaría exactamente industrialización.

Sin embargo, no fue esta elite agraria o sus representantes los que introdujeron los proyectos de modernización de la agricultura en aquel estado. Irónicamente o no, sería otra tendencia - más urbana e industrial que tendría en João e Israel Pinheiro y Juscelino Kubitschek expresión de destaque -, la responsable de iniciar e intensificar este proceso. Estos políticos tendrían la conciliación con las elites agrarias, la base para la implementación de un modelo de modernización industrial en Minas Gerais. João Pinheiro, presidente de la provincia de Minas Gerais por un pequeño período en 1890 y entre 1906 y 1908, “aunque se haya llegado a un acuerdo con los propietarios rurales y se haya basado parte de sus propuestas en la modernización de la agricultura, a través del uso de nuevas tecnologías, traía también planes de industrialización." (Carvalho, 2005, p. 68) Así, João Pinheiro, que tenía como interés principal el proceso de industrialización, se convirtió en un referente en los procesos de modernización de la agricultura.

Después de caracterizar el período como "crítico", principalmente por la interpretación de estancamiento económica del estado, João Pinheiro organizó en 1903 el Congreso Agrícola, Industrial e Comercial. Así, la idea de recuperación económica fue desarrollada por las elites mineiras con la intención de emprender un plan de desarrollo económico y social, buscando evidenciar que la provincia de Minas Gerais había declinado económicamente después del ciclo del oro. El siglo XIX, entonces, fue interpretado por el concepto de "estagnación" (Dulci, 1999, p. 39) En las décadas de 1930 y 1940 esta idea fundamentó las acciones de la política económica, Benedito Valadares, interventor nombrado por Getúlio Vargas entre 1933 y 1945 "fue responsable, en materia de política económica, del inicio de un cambio definitivo de Minas hacia la industrialización". De esta forma, después de la crisis de 1929 "la provincia tenía que encontrar nuevas fuentes de riqueza, la misma preocupación de João Pinheiro" (Carvalho, 2005, p. 69) y el Congreso de 1903. Valadares tuvo en su administración el apoyo del ingeniero Israel Pinheiro, hijo de João Pinheiro. Israel inició su carrera política a nivel provincial cuando asumió la Secretaria de Viação, Obras, Agricultura, Indústria e Comércio de Minas Gerais, nombrado por Valadares en 1934. Israel Pinheiro fue responsable de la división de esta secretaría - una de Viação e Obras Públicas y otra de Agricultura, Indústria e Comércio - y posteriormente organizó y presidió la Companhia Vale do Rio Doce, invitado por Getúlio Vargas en 1942 (Pinheiro Filho, 2005, pp. 8-9).

De acuerdo con Otávio Dulci, la búsqueda de nuevas fuentes de recursos y las necesidades de desarroIlo "fueron inspiradas por dos imágenes distintas de la economía de la región y su integración al sistema nacional. Inicialmente dominado, el diseño de una economía altamente diferenciada con una agropecuaria fuerte como base de una industria que se quería agilizar". La idea de especialización productiva ganó importancia en un segundo momento, cuando "los esfuerzos se centraron en la industria en expansión, y dentro de este, en el sector de bienes intermedios" (Dulci, 1999, p. 38).

De esta forma, si el gobierno del interventor Valadares influyó en el proceso de industrialización, un antiguo opositor de Getúlio Vargas y elegido gobernador por la União Democrática Nacional (UDN), Milton Campos, dio continuidad a este proceso cuando en su gobierno fue elaborado el "Plano de Recuperação Econômica e Fomento da Produção", en 1947. La iniciativa del gobierno Milton Campos se tornó "la primera experiencia de macroplanificación en escala regional en Brasil". Este plan, "por sus orientaciones centrales, sobre todo el énfasis en la modernización equilibrada de la industria y de la agricultura, la traducción de lo que se podía llamar modelo udenista de modernización, en contraste con el modelo pessedista, que se realiza a continuación con Juscelino Kubitschek" (Dulci, 1999, p. 78).

Si el argumento de Dulci distingue los modelos de desarrollo udenista y pessedista, la creación de la ACAR representó una continuidad entre el gobierno de Milton Campos (UDN - 1947-1950) y de Juscelino Kubitschek (PSD - 1951-1955), que nacionalizó el programa durante su mandato como presidente (19561961). Según el agrónomo y extensionista José Paulo Ribeiro, el responsable de la continuidad del programa fue João Napoleão de Andrade que había convencido al gobernador Juscelino Kubitschek: "al comienzo de su mandato, el gobernador Kubitschek, influenciado por personas o grupos, estaba por decidir si terminaba o no con la ACAR". De esta manera, "antes de tomar la decisión final llamó al Palácio da Liberdade a su 
amigo de partido John Napoleón Andrade, con amigos en los Estados Unidos, explicándole lo que pensaba y solicitando que el mismo estudiase el tema." Durante la Guerra, Andrade estuvo en los Estados Unidos, ya que su familia era exportadora de cristal de roca y, "durante su estadía en aquel país, tuvo la oportunidad de conocer el servicio de extensión rural y otros servicios de apoyo a la agricultura, pues, como hacendado en el municipio de Sete Lagoas, estaba interesado en el tema." Ante las consideraciones del emisario João Napoleão de Andrade, Kubitschek sería no solo seguido con el programa ACAR, sino también apoyando totalmente la iniciativa (Ribeiro, 2000, p. 101).

Más que una iniciativa aislada, es necesario pensar que la permanencia de la ACAR se conciliaba con los proyectos de las dos corrientes políticas. Esto porque la agencia minera evidenciaba un tono conciliador entre los dos proyectos: si para los udenistas los programas de la AIA desarrollados en Minas Gerais representaban la modernización de la agricultura en una relación que podría volverse más empresarial, en el sentido de que el productor rural pasaría cada vez más a diversificar la producción, para los pessedistas estos programas no influirían en la estructura agraria. El programa extensionista nació de la relación con los pequeños agricultores, en la década de 1960, por ejemplo, la Extensión Rural pasa a ser utilizada como instrumentos de los grandes propietarios.

\section{IMPACTOS PARA LOS AGRICULTORES Y REORIENTACIONES}

Mientras que la noción de industrialización se articulaba a la agricultura, el medio rural todavía era caracterizado por la ACAR como un espacio a ser modificado. Así, en la década de 1950, con el inicio de los trabajos extensionistas, "el hombre rural" era interpretado como "retraído y de naturaleza sospechosa, aislado en su medio ambiente por la falta de medios de comunicación y de intereses comunitarios". Este hombre "ha vivido al margen del progreso agrícola". Si la ACAR diagnosticó el medio rural de tal forma, esas condiciones, de todo modo, serian culturales y, por lo tanto, posibles de sufrir modificaciones. En esta línea se concebía que, "por una reacción psicológica natural, [el agricultor] no se apresuraba en abandonar los métodos de trabajo de rutina y anticuados". Y eso no solo se daba apenas por una resistencia natural, sino también "por la deficiencia de los métodos de orientación y enseñanza que se han aplicado a las zonas rurales" (ACAR, 1959, p. 48). Se ha evaluado, entonces, que con métodos adecuados, "nuestro hombre rural" cada vez más se ha "demostrado dispuesto a avanzar y ampliar sus conocimientos". Así, el "hombre rural quiere progreso", sentenciaría un informe del ACAR (ACAR, 1957, p. 3).

Fueron muchos los intentos de hacer que el agricultor mineiro "progresara". Ajustar estos métodos de trabajo y tecnología sería de fundamental importancia para que el hombre del campo alcanzara estos objetivos. Mientras que al principio de los años 1950 el crédito y la asistencia técnica constituían el principal modelo de actuación, supervisando la atención de pequeños propietarios, a fines de aquella década los objetivos eran más osados: la "educación tecnológica impulsa el progreso" (ACAR, 1960, s.p.), o sea, a través del trabajo de las agencias como la ACAR podría ser posible alcanzar determinados objetivos económicos y sociales. Por ello, el término "progreso" significaría un estado ideal a ser alcanzado, es decir, "zonas rurales totalmente desarrolladas, habitada por gente educada con un nivel de vida razonable" (ACAR, 1959, p. 48), necesitando acabar con posibles trabas: "a veces, el aumento se logra por los agricultores en la producción de cultivos [...], a veces la satisfacción de una ama de casa cuyos hijos tienen salud y apariencia, a veces una mejor vestimenta, hecha por manos una vez inhábiles y rudas, hoy ágiles y caprichosa, ahora la construcción de una vivienda en sustitución a una cafua aún indigna hasta de este nombre" (ACAR, 1959, p. 48).

Les cabía a los agentes de la ACAR "la introducción en el medio rural de las prácticas modernas de agricultura y economía doméstica" y esto implicaría "la adopción de equipos y elementos a veces desconocidos" de parte de los agricultores. Teniendo en cuenta que no habían elementos disponibles o que los mismos eran considerados muy costosos, la ACAR entonces "se compromete en comprar en las fuentes de producción, otorgándoselo a los agricultores a un bajo costo" (ACAR, 1959, p. 5). Los principales productos eran filtros de agua, fertilizantes químicos, semillas seleccionadas, insecticidas, pulverizadores y máquinas agrícolas. Con el espiritu de superar el aislamiento de las poblaciones rurales, la ACAR trató de introducir una serie de actitudes objetivando el desarrollo de la agricultura. Teniendo como objetivo acercar a los agricultores a las propuestas de la ACAR en este período, la manera adoptada para iniciar un proceso de cambio fue la introducción de elementos técnicos y/o tecnológicos también para los jóvenes, que serían considerados elementos de conducción del proceso de cambio social (ACAR, 1959, p. 19).

De acuerdo con la propuesta de la ACAR, fue necesario articular un trabajo que integrara la Extensión 
de las áreas de la salud y educación (universidades y escuelas) y que fuera realizado en colaboración con los gobiernos municipales, provinciales y nacionales. En otras palabras, si en las relaciones "macro" la gran cuestión estaba en como vencer las resistencias políticas para iniciar y legitimar el trabajo, como fue el caso del SAPS o de las experiencias paulistas, en un nivel "micro", lo que se pondría en escena eran las discusiones sobre cómo conducir un proceso de cambio social. "Se trata de un personal autorizado para ello", por ende, "consciente de su misión que lleva a las familias rurales de los logros modernos de la ciencia y de la técnica, la investigación y la experimentación en el campo de la agricultura y de la economía doméstica" (ACAR, 1959, p. 2).

La que se tornó símbolo del trabajo extensionista de la ACAR seguía el modelo norteamericano y era constituida por dos agentes, generalmente un técnico agrícola o ingeniero agrónomo y una persona con instrucciones en economía doméstica, teniendo en consideración la dificultad en contratar profesionales con formación en Economía Doméstica en Brasil. Sumado a eso, estaba el famoso Jeep: "dos extensionistas y un jeep" era la frase que demostraba un ideal para los extensionistas, por encima de cualquier otra cosa. La relación propiedad-hogar estaba contemplada en las actividades específicas de los extensionistas y ellos eran llevados a las lejanas propiedades rurales con el auxilio del Jeep Willys, muy utilizado en la Segunda Guerra Mundial.

Ese modelo experimentado a lo largo de la década de 1950 - con contornos fuertemente civilizadores, tanto por la tradición del FSA como del extensionismo - y con profundas alteraciones en las décadas siguientes, no fue aceptado sin resistencias. Aunque a nivel provincial la AIA contase con el apoyo del gobierno mineiro para la creación de la ACAR, a nivel local las relaciones se establecieron de formas variadas. Son muchos los ejemplos de que el trabajo fue bien recibido por entidades representativas de los productores rurales, por políticos y por la Iglesia Católica. No obstante, con la apertura de diversas oficinas cubriendo gran parte del estado de Minas Gerais, aun en la década de 1950, el poder local también marcó el compás de las actividades de crédito y extensión rural.

Inicialmente, la profesión de ingeniero agrónomo en Brasil se formó bajo un clima de "apoliticismo" y "neutralidad", ubicándose como clase reunida en torno de los ideales de modernización de la agricultura. Aún en el inicio del siglo XX, la opción de los agrónomos para la aplicación de los conocimientos considerados modernos, no estaba hecha para una pequeña propiedad. En la segunda mitad del siglo $X X$, ese modelo se ajustó a las nuevas necesidades de producción y mantenimiento de la estructura agraria. Así, emergen diferencias que apuntan cada vez más al argumento de que los programas agrícolas no fueron simples modelos importados, pero, reformulados. Un ejemplo de ello, es que el trabajo extensionista en los Estados Unidos estuvo cada vez más vinculado a la intensificación de la capacidad productiva de determinadas áreas - como en el ideal norteamericano de transformar el desierto en jardín - sobre todo de pequeñas propiedades afectadas por la erosión, evento muy común, principalmente después de 1929. La aplicación de los ideales extensionistas en Brasil, mientras tanto, buscó adaptarse a las condiciones locales de la estructura agraria.

Solo al final de la década de 1950 el tema de la reforma agraria ha sido introducido en ACAR, posiblemente porque eran tiempos en que esa discusión tomó formas irremediables a nivel nacional, principalmente en los años que antecedieron el golpe militar de 1964. Si en 1961 el Presidente de la República invitó a los "eminentes patricios" de la ACAR, para unirse al ex gobernador y entonces senador Milton Campos, a hacer parte de una comisión de trabajo para estudiar el Estatuto da Terra (Libro de Actas, 1961, s.p.) la ACAR poco se involucró para modificar la estructura agraria en el periodo estudiado. Por otro lado, también sería equivocado pensar que, en este periodo, la actuación de la agencia fue vinculada a los latifundistas, lo que fue, de hecho, más frecuente después de 1964, bajo el control de los militares sobre el sistema ABCAR.

Es posible afirmar que, en la década de 1950, el poder local trató de ajustarse al programa de Crédito y Extensión Rural a las condiciones históricamente establecidas en determinadas comunidades. En otras palabras, la ACAR y los representantes del conocimiento técnico, en contacto con grupos políticos, económicos y religiosos, necesitaron negociar para legitimarse y hacer efectivo sus programas de acción. De acuerdo con el antropólogo norteamericano Charles Wagley, Minas Gerais era caracterizada por ciudades con una población de menos de cinco mil habitantes, lo que favorecía el prestigio religioso, fruto de su formación histórica (Wagley, 1965).

Primeramente, la ACAR estableció una disciplina rígida sobre sus técnicos. Actuar políticamente era prohibido a todos los extensionistas, bien como otras reglas eran destinadas exclusivamente a las mujeres: vestir pantalones largos, no fumar o incluso casarse. 
La religiosidad no se constituyó en regla, pero, también provocó conflictos, como se verá adelante. Los mecanismos de control ejercidos fueron introducidos por los norteamericanos. Según la psicóloga Rita Hilarina Gomes Nelson, "ellos eran muy exigentes, querían que todo estuviera [hecho] bien. Cuando trabajábamos, nosotros íbamos a las propiedades y no podíamos aceptar almuerzo ni regalos, eso era prohibido. No podíamos involucrarnos con política, era apenas trabajo mismo" (De Paula, 2005, p. 26).

Los representantes locales no dejaron de auxiliar el trabajo de la ACAR. El alcalde de la ciudad de Poté, João Ferreira de Oliveira, por ejemplo, "fue [considerado] el alcalde más progresista, [pues] abrió varias carreteras con máquinas para que la ACAR diera asistencia a los productores." (Projeto Memória, 2005b, p. 5). Otros alcaldes contribuyeron con muebles para diversas oficinas. La conciliación marcaría las relaciones políticas en determinadas localidades, como en la municipalidad de Candeias. El alcalde de la ciudad, José Pinto de Resende, miembro de la UDN, "transfirió todo el poder de apoyo al servicio de la ACAR al concejal William Viglioni, entonces perteneciente a la bancada del Partido Trabalhista Brasileiro (PTB), partido de oposición al Alcalde Municipal" (Projeto Memória, 2005c, p. 5). Nestor Lamounier, ex concejal en la época de instalación de la oficina del ACAR (1955), narró que los concejales "tuvieron que votar un proyecto para liberar recursos destinados a la adquisición de los muebles para la oficina de la ACAR. El proyecto fue aprobado por unanimidad por la Cámara." De esta forma, aún de acuerdo con Lamounier "iniciaba allí, con la instalación de la ACAR, el progreso del municipio." (Projeto Memória, 2005c, p. 5)

En Santa Lúcia, por ejemplo, la ACAR realizó acuerdos con la municipalidad y comerciantes locales, pretendiendo solucionar el problema de una familia que no tenía acceso al agua potable en su propiedad, desarrollando parásitos: "el compromiso del Ayuntamiento [sería] dar la mano de obra para captación del agua" (Projeto Memória, 2005d, p. 1). En 1951, en la comunidad de Martins Guimarães, la relación entre la ACAR y la Iglesia Católica era marcada por la colaboración de curas. De acuerdo con la narrativa de un ingeniero agrónomo no identificado:

Había sólo unas pocas casas y una iglesia y la población local no aceptaba la ACAR porque pensaba que era comunista y que podía llevarles cosas de la población de los Estados Unidos. Tenían prejuicios en mezclar frutas con leche. Así que, con la ayuda del cura Guarino, la ACAR hizo una ensalada de fru- tas y después de una misa el cura "obligó" la gente a comer la ensalada de frutas y beber la leche. La población con miedo del cura comió la ensalada sin lugar a dudas y acabó disfrutando y perdió el miedo a la mezcla de frutas. Con esto comenzó a disfrutar de todos los frutos que se estaban desperdiciando (Projeto Memória, 2005a, p. 1).

Por otro lado, no faltaban ejemplos de que muchos conflictos surgieron de la relación entre la política local y la ACAR. Amenazas de desalojo, cambio de local o de mobiliario sacado, cedido por la municipalidad a la oficina de la ACAR hicieron parte del repertorio. En el municipio de Poté, la oficina local fue inaugurada en 1955. El extensionista Odelson Gomes da Silva, más conocido como Deca, relató que "para el cargo de auxiliar de oficina [de la ACAR] hubo un concurso local, y él fue el único que aprobó. Como era adversario político del Alcalde Omar Afonso, este le dijo que si tomase posesión, llevaría los muebles de la oficina de ACAR" (Projeto Memória, 2005b, p. 1). Se cuenta, sin embargo, que el alcalde no realizó tal acción.

Volviendo a los documentos de la época, encontramos en un periódico local del pequeño municipio de Paraguassú una noticia bastante curiosa, publicada en 1959. La noticia se había apurado en decir que la ACAR no cerraría su oficina en el municipio, "Podemos asegurar que es absolutamente infundado el rumor de que la ACAR cerrará su oficina en esta ciudad por falta de interés de nuestras autoridades y de la gente por sus servicios." (Jornal O Paraguassú, 1958, s.p.) Noticia curiosa que al negar, casi confirma los conflictos latentes. No se tiene la posesión de documentos sobre los desdoblamientos posteriores en la ciudad. No obstante, son indicios de la complejidad de relaciones entre los representantes y la ACAR, la sociedad y el poder local. Esa complejidad exigió que, en diferentes municipios, muchas de las acciones fueran reorientadas.

Cuando en el trabajo de campo, objetivando la legitimación frente a los agricultores, los extensionistas necesitaban lidiar con resistencias políticas, religiosas y de aquellos a quien requerían asistencia técnica, los agricultores. Caracterizado como un estado religiosamente conservador, la figura del cura ejercía gran influencia en el medio rural mineiro, conforme ha señalado por el antropólogo norteamericano Charles Wagley.

En enero de 1954, en el municipio de Machado, el cura Pedro Strabelli fue informado acerca de que la extensionista Zélia Rodrigues, que actuaba en la comunidad, era protestante de la iglesia presbiteriana. En una 
demostración de poder sobre "su" comunidad, el cura realizó la denuncia a la ACAR, pidiendo que la misma fuera despedida por no estar de acuerdo con los preceptos religiosos del local. De acuerdo con el ingeniero agrónomo Domingos Machado, "el cura reconocía el buen trabajo" de Zélia Rodrigues, "pero no admitía su presencia en el municipio por ser presbiteriana". En ese sentido, Strabelli "pidió a la ACAR la dimisión de la referida empleada y puso la población católica en contra [ella], creando un clima insoportable para el trabajo. Para solucionar el problema, [Zélia] fue transferida a fines de marzo de 1954" (De Paula, 2005, p. 25). Aun sin despedir una de sus más expertas funcionarias, la ACAR necesitó ceder, pues necesitaba del apoyo de la Iglesia Católica para realizar sus actividades.

El extensionista Aldo Borges, colega de Zélia Rodrigues, concurrió, por la UDN, al puesto de concejal en el mismo municipio de Machado, contrariando, en realidad, las orientaciones de la ACAR de "no involucrarse con política". Cuenta la historia que la candidatura del extensionista causó verdadera "convulsión social", una vez que la ACAR se vio vinculada en la disputa política histórica entre dos familias tradicionales de la región. Según el relato de la época, "el hecho se agrava por disputas políticas entre la UDN y el PSD, que se convirtió en pelea de familia, dividiendo la población de la ciudad: por un lado, el alcalde Gustavo Carneiro Dias, el hermano de la secretaria de la oficina local y jefe de la UDN y por otro, por el PSD, Felicitando Dr. Vieira, colega y amigo del Gobernador y cuñado de la secretaria de la oficina local." (De Paula, 2005, p. 25-26)

Ese conservadorismo político de grupos locales parece estar latente en el conflicto afrontado por el extensionista de la ACAR. Sus funcionarios buscaron formas de solucionar la cuestión. Según el extensionista Geraldo Machado, como forma de evitar conflictos más grandes, la Polícia Militar de las ciudades cercanas fue reunida para intervenir en el conflicto: "el clima político era insoportable y para mantener el orden público el Batalhão da Polícia Militar, con sede en Lavras, fue transferido para el municipio. La situación llegó a tal punto que el Supervisor Regional cuya oficina tenía su sede en Varginha, no pudo ir más a Machado, con lo cual la oficina no sigue la filosofía de la ACAR." (De Paula, 2005, p. 25). Aun con la intervención directa de la ACAR, en el momento en que el Directorio se resolvió por la dimisión de Aldo Borges, el acuerdo político entre el entonces gobernador Juscelino Kubitschek con la AIA enfrentó una serie de problemas en función de este episodio.
Así, "después de larga discusión sobre el tema por el Director de la AIA, Mr. Crawford, que vino de los Estados Unidos y el Gobernador de la Provincia, el problema ha sido resuelto, manteniendo la oficina funcionando" con dos nuevos supervisores (De Paula, 2005, pp. 25-26).

Desde la mitad de la década de 1950, la AIA insistía en demostrar la intención de dejar la dirección de los trabajos bajo el dominio de los brasileños, pero, en igual medida, percibían que a ellos les gustaría que los norteamericanos siguieran conduciendo la ACAR: "los miembros brasileños de la ACAR se niegan a admitir a si mismos que AIA nunca va a dejarles que hagan el trabajo de ACAR (From Walter Crawford to Louise Boyer, 1958, p. 3). La justificación para Juscelino Kubitschek, y otros que también pensaban de esa forma era la de que la ACAR, con los norteamericanos en la dirección, estaría distante de las cuestiones políticas, lo que le daría un aire de "neutralidad". En la percepción de Harry Bagley, Juscelino Kubitschek "y otros líderes de Minas siempre han insistido en que la manera de mantener la ACAR fuerte, y hacerla más fuerte, es dejar la dirección actual en manos de la AIA [...] [que] se ejecutará la ACAR como un programa técnico, separado de la política" (Excerpts from letter, 1955, s.p.).

Luego, un grupo de brasileños tenía la intención cada vez más fuerte de asumir la responsabilidad. El sustituto de Crawford, el norteamericano de Nuevo México, Santiago Apodaca tenía una relación conflictiva con el extensionista brasileño Geraldo Machado en función de la diferencia de concepción de administración del ACAR. Fue exigido, mediante los conflictos, la reorganización de las actividades: Apodaca fue transferido para el ABCAR en 1956, como jefe de la Subdivisión Técnica, permaneciendo como miembro de la Junta Administrativa, pero no en el cargo de Director Ejecutivo, ocupado ahora por Crawford. Geraldo continuaría como Director Asociado de la ACAR, pero teniendo Crawford y Bagley como una especie de superiores directos, con la intención de ser oídos antes que cualquier decisión fuera tomada. (From Walter Crawford to Louise Boyer, 1958, p. 3) En la opinión de Walter Crawford, "Machado ha demostrado ser deficiente en habilidades directivas y en los aspectos de las relaciones humanas del trabajo" en el momento en que "existe una tendencia aislacionista de los mejores técnicos de ACAR lejos de ABCAR, otros programas estatales y la orientación técnica extranjera" (From Walter Crawford to Louise Boyer, 1958, p. 3). 


\section{CONCLUSIONES}

El presente texto discutió cómo la actuación de la AIA en Brasil se caracterizó por un intenso proceso de negociación entre 1946 y 1961. Esa agencia heredó un know-how aun en los tiempos de la Segunda Guerra, con el trabajo del Office (la Oficina) y amplió gradualmente sus proyectos y su área de actuación tanto en Brasil como en Latinoamérica, y otros países durante los veinte y un años de funcionamiento de la agencia. Contemporánea a la emergente ideología de la modernización, la AIA buscó detectar cuales eran los posibles proyectos adecuados para la América Latina, en especial Brasil y Venezuela. Inspirada en las experiencias norteamericanas de crédito rural, el extensionismo, entre otras estrategias de desarrollo, esa agencia trató de adaptar dichos modelos a las condiciones locales, teniendo en cuenta que, en la visión de la propia AIA, los proyectos que tuvieron éxito en los Estados Unidos también lo tendrían en los países en desarrollo, siempre y cuando fueran adaptados. Proyectos que involucraron intensos debates (FSA) o un largo proceso de consolidación (Extension Service) en aquel país fueron vistos entonces como una manera de abrir los caminos para alcanzar objetivos semejantes de cambio social, económico y político.

Con eso, la transformación de las sociedades latinoamericanas en naciones democráticas, liberales y de economía capitalista auxiliaría en el mantenimiento o en la ampliación de los negocios económicos y políticos norteamericanos, protegiendo tales intereses de la creciente "amenaza comunista". Los debates internos en los Estados Unidos apuntaban hacia una posible depresión debido a las altas tasas de producción agrícola durante el período de la guerra y por consecuencia, la apertura de nuevos mercados y la consolidación internacionalista en forma de cooperación internacional podría dar un nuevo aliento para la economía norteamericana. Nelson Rockefeller sería indicado como uno de los responsables del mantenimiento y de la ampliación de los intereses económicos y políticos en Latinoamérica, aun con el cierre del Office. Desde esa perspectiva, uno de los instrumentos para la continuación de ese proyecto sería la constitución de una agencia filantrópica, la AIA. Por otro lado, no se puede dejar de notar que la confianza en la ciencia y la tecnología hace parte del mismo proyecto, en el cual filantropía y difusión de conocimientos técnicos funcionan como sinónimos, y, por lo tanto, se diferencian de prácticas colonialistas.

Sin embargo, tratando de interpretar el proceso no solo como la imposición de determinados proyectos de la AIA para el país, se busca demostrar que los procesos de negociación para la implementación de determinados programas fueron intensos: mientras algunos proyectos recibían mayor apoyo de instituciones o grupos políticos, otros no se desarrollaban de la manera esperada, indicando aquí la importancia de la participación de los diferentes grupos políticos brasileños en el "éxito" o no de un determinado programa.

Por ende, si la AIA encontraba apoyo gubernamental para la fundación de una agencia de crédito y asistencia técnica como el ACAR en Minas Gerais, por otro lado, programas como el SVA o incluso la experiencia paulista no ejerció la influencia deseada frente a los gobiernos federal y paulista respectivamente. Con eso, al iniciar la década de 1950, la AIA desarrollaba de forma intensificada los programas en Brasil: uno en el interior de la provincia de São Paulo, pautado en el extensionismo rural, y otro en Minas Gerais, con influencia de trabajos en crédito bajo la supervisión de la Farm Security Admistration. Aunque los programas paulistas tuvieron una buena aceptación al principio, los grupos políticos del estado prefirieron apoyar el mantenimiento de otros programas entonces existentes como la Casa da Lavoura. En 1956 el programa fue cerrado. En Minas Gerais, por otro lado, el gobierno del estado estableció relación con la AIA para la creación de la ACAR, y aun con dificultades iniciales en la excepción, el programa fue amparado por los grupos políticos justamente por estar en consonancia con los ideales de industrialización en el periodo.

Sin embargo, la estrategia de modernización de la producción agrícola en Minas Gerais presentó resistencias a nivel local, cuando de la ampliación de las oficinas de la ACAR: políticos tradicionales o curas se relacionaron de forma conflictiva, aunque en otras ocasiones los mismos actores locales legitimaron el trabajo de la agencia de crédito y asistencia técnica. Además, los "paquetes" pensados originalmente no obtuvieron el éxito esperado, como en la ocasión en que los candidatos a mutuarios de la ACAR presentaron un perfil totalmente distinto al pretendido por la agencia y que, sumándose a las dificultades de liberación de crédito para los productores rurales, llevaron la ACAR a la estrategia de "matar hormigas" para "crear buenas relaciones". La expansión del modelo de la ACAR para otras provincias de Brasil y la creación de la ABCAR involucró un proceso conflictivo con agencias formadas bajo la influencia del Punto Cuatro - del cual contradictoriamente Nelson Rockefeller era uno de los representantes, como el ETA, y casi excluyó la AIA del proceso de fundación de la agencia nacional. 


\section{AGRADECIMIENTOS}

Agradezco a las siguientes personas e instituciones por hacer posible la elaboración de este artículo: Casa de Oswaldo Cruz, COC/Fiocruz e EMATER en Brasil, el

\section{NOTAS}

1 El programa de asistencia técnico financiera a los países considerados subdesarrollados, Ilamado Punto Cuatro (Point Four), fue originado del cuarto punto del discurso inaugural del Presidente Harry Truman 1949 y entró en acción en 1950. La Extensión Rural, uno de los programas desarrollados por la AIA en América Latina, por ejemplo, también fue uno de los principales puntos de la cooperación técnica durante los tiempos de la Alianza para el Progreso.

\section{FUENTES PRIMARIAS}

American International Association for Economic and Social Development. A statement of organization, activities and objectives. Jan. 1949. Collection AIA, Box 10, Folder 85.

Excerpts from letter of October 20, 1955 from Harry W. Bagley, AIA representative in Brazil. Collection: Family, Record Group: III 4B, Box 01, Folder 07 AlA - Brazil.

From Henry Bagley to Dick Greenebaum. Subject: AIA in state of São Paulo. 01/28/1955. Collection: Family, Record Group: III 4B, Box: 01, Folder 07, AIA Brazil.

From Walter Crawford to Louise A. Boyer. Confidential. Brazil Program. 08/08/1958. Collection: Family, Record Group: III 4B, Box: 29, Folder 283.

\section{BIBLIOGRAFÍA}

Adas, Michael (2003), "Modernization theory and the American revival of the scientific and technological standards of social achievement and human worth". En: Engerman, David C.; Gilman, Nils; Haefele, Mark H; \& Latham, Michael (ed.), Staging Growth. Modernization, development, and the Global Cold War, Amherst, University of Massachusetts Press.

Andrade, João Napoleão de (1982), Entrevista. Belo Horizonte, Emater/MG.

ASSOCIAÇÃO de Crédito e Assistência Rural de Minas Gerais/ACAR (1957), ACAR. Relatório 1956/57. Belo Horizonte, ACAR.

ASSOCIAÇÃO de Crédito e Assistência Rural de Minas Gerais/ACAR (1959), ACAR. Dez anos a serviço do povo rural de Minas Gerais. Belo Horizonte, ACAR.
Rockefeller Archive Center en los Estados Unidos. Gracias a Robert Wegner por la orientación de la tesis de doctorado de la cual resulta este artículo.

2 Servicio de Alimentación de la Seguridad Social.

3 Asociación de Crédito y Asistencia Rural.

4 Como escribió Cotta (2008, p. 45), el Estado de São Paulo tenía, en $1947,268.240$ hacendados, donde 243.990 eran pequeños propietarios con menos de 50 alqueires paulistas, siendo que entre estos 103.572 poseían menos de cinco alqueires paulistas

From Walter Crawford to Wallace Harrison. Subject: Renewal and extension of the AIA São Paulo agreement. p. 01. 05/27/1954. Collection: Family, Record Group: III 4B, Box 01, Folder 7, AIA Brazil.

JORNAL O Paraguassú. Paraguassú, Minas Gerais, 02/08/1959.

Libro de Actas de la Yunta Administrativa de ACAR 1959-1970. Acta de la sexagésima cuarta reunión de la Yunta Administrativa de la Associação de Crédito e Assistência Rural (ACAR). Belo Horizonte, 13 de mayo de 1961.

The American International Association for Economic and Social Development. An explanation of its objectives and method of operation by Kenneth J. Kadow for Nelson A. Rockefeller. 02/19/1946. Collection: Family. Record Group: III 4 B. Box 01, Folder AIA - Simon Bolivar Foundation. p. 14.

ASSOCIAÇÃO de Crédito e Assistência Rural de Minas Gerais/ACAR (1960), ACAR. Programa para o ano agrícola 1959/60. Belo Horizonte, ACAR, s.p.

Carvalho, José Murilo de (2005), “Ouro, terra e ferro. Vozes de Minas". En: Gomes, Ângela de Castro (Org.), Minas e os fundamentos do Brasil moderno, Belo Horizonte, UFMG.

Cotta, Luiza Cristina Villaméa (2008), Adhemar de Barros (19011969): a origem do "rouba, mas faz". São Paulo, USP, p. 45 (Dissertación de Maestria em História Econômica).

Dalrymple, Martha (1968), The AIA Story: two decades of International Cooperation. New York, AIA.

Dulci, Otávio Soares (1999), Política e recuperação econômica em Minas Gerais. Belo Horizonte, UFMG. 
Escobar, Arturo (Entrevista), (1995), Encountering development. The making and unmaking of the third world. Princeton, N.J., Princeton University Press.

Latham, Michael (2003), "Introduction: modernization, international history, and the Cold War World". En: Engerman, David C.; Gilman, Nils; Haefele, Mark H; \& Latham, Michael (ed.), Staging Growth. Modernization, development, and the Global Cold War, Amherst, University of Massachusetts Press.

O'Brien, Thomas F. (1996), The revolutionary mission. American enterprise in Latin America, 1900-1945. New York, Cambridge University Press.

Paula, José Alfredo Amaral de (2005), Fatos históricos da ACAR pesquisados pelo Eng. Agr. José Alfredo Amaral de Paula. Belo Horizonte, EMATER, Texto Inédito.

Pinheiro Filho, Israel (2005), "Prefácio". En: Gomes, Ângela de Castro (Org.), Minas e os fundamentos do Brasil moderno, Belo Horizonte, UFMG.
PROJETO MEMÓRIA (2005a), Fatos pitorescos sobre a ACAR em Lagoa da Prata. Belo Horizonte, EMATER, Texto Inédito.

PROJETO MEMÓRIA (2005b), 40 anos de extensão rural em Poté. Belo Horizonte, EMATER, Texto Inédito.

PROJETO MEMÓRIA (2005c), Escritório Local de Candeias. Belo Horizonte, EMATER, Texto Inédito.

PROJETO MEMÓRIA (2005d), Escritório de Santa Luzia. Belo Horizonte, EMATER, Texto Inédito.

Reich, Cary (1996), The life of Nelson A. Rockefeller. Worlds to conquer. 1908-1958. New York, Doubleday.

Ribeiro, José Paulo (2000), A Saga da Extensão Rural em Minas Gerais. São Paulo, Annablume; Minas Gerais, CPP/Emater.

Wagley, Charles (1965), An introduction to Brazil. New York, Columbia University Press. 